\title{
Towards improved Hashin-Shtrikman bounds on the effective moduli of random composites
}

\author{
SÉBASTIEN BRISARD ${ }^{\mathrm{a}}$ \\ Université Paris-Est, Laboratoire Navier, ENPC, IFSTTAR, CNRS UMR 8205, 6 et 8 avenue Blaise Pascal, \\ 77455 Marne-la-Vallée Cedex 2, France
}

Received 7 December 2015, Accepted 4 July 2016

\begin{abstract}
The celebrated bounds of Hashin and Shtrikman on the effective properties of composites are valid for a very wide class of materials. However, they incorporate only a very limited amount of information on the microstructure (volume fraction of each phase in the case of isotropic microstructures). As a result, they are generally not tight. In this work, we present an attempt at improving these bounds by incorporating explicitly the local volume fraction to the set of local descriptors of the microstructure. We show that, quite unexpectedly, the process fails in the sense that the classical bounds are retrieved. We further show that this negative result applies to so-called weakly isotropic local descriptors of the microstructure (to be defined in this paper). This suggests that improved bounds may be obtained with anisotropic descriptors.
\end{abstract}

Key words: Elasticity / homogenization / bounds / effective properties / local volume fraction

\section{Introduction}

Bounds on the effective properties of composites are very useful tools, as they provide exact safeguards for more elaborate estimates. Among all available bounds, those of Hashin and Shtrikman are probably the most useful, as they only require the volume fractions of the phases, and apply to a wide class of composites (namely, isotropic microstructures). The price to pay for this simplicity and generality is, of course, the fact that these bounds are usually relatively slack. That they are insensitive to relative sizes of the inclusions constitutes another major shortcoming.

Sharper bounds have been produced, which improve on the bounds of Hashin and Shtrikman; see e.g. [1]. However, they generally involve complex statistical descriptors of the microstructure which are difficult to measure. Besides, it is not possible to choose these statistical descriptors, as they merely are an outcome of the whole optimization process.

In this paper, we present an attempt at improving the classical bounds of Hashin and Shtrikman. To do so, we carry out the same optimization process as in the classical approach, with an enriched trial field. This is a potentially very flexible approach, since any local descriptor

\footnotetext{
${ }^{a}$ Corresponding author:

sebastien.brisard@ifsttar.fr
}

can be used as enrichment. As a first step, we use local volume fractions as supplementary local descriptors of the microstructure. This was suggested by previous work by Widjajakusuma et al. [2], and by the fact that such descriptors effectively introduce a length-scale (the size of the sliding window). The resulting bounds were expected to be sensitive to the relative size of the inclusions.

The somewhat unexpected outcome of this approach is the fact that the resulting bounds coincide with those of Hashin and Shtrikman. In other words, the supplementary microstructural information was ignored by the optimization process. We were able to extend this negative result to the class of weakly isotropic local descriptors of the microstructure, that will be defined more precisely below. This now suggests to explore the class of anisotropic local descriptors.

The present paper is organized as follows. The improved bounds on the macroscopic properties of composites that we seek in this work are derived by means of polarization techniques within the framework of linear elasticity. In Section 2, we provide a brief account of these techniques; in particular, we introduce the energy $\mathcal{H}$ of Hashin and Shtrikman [3] (see also [4] for a modern presentation). In Section 3, we construct enriched trial fields which incorporate supplementary local descriptors of the microstructure. We then carry out the optimization process presented in Reference [4] to derive bounds of the macroscopic properties, and show that these bounds 
fail to improve on the classical bounds of Hashin and Shtrikman [5]. This negative result is then extended to weakly isotropic local descriptors of the microstructure. Section 4 closes this paper with a few thoughts on how to overcome the limitation highlighted in Section 3.

It should be noted that this paper makes use of the classical terminology of apparent stiffness and statistical volume element (SVE) [6,7].

\section{Polarization techniques for linear elasticity}

The standard presentation of these techniques requires the use of the Green operator for strains of a bounded domain, which is generally unknown. Following Willis [4], it is usually replaced with the Green operator for strains of the whole space $\mathbb{R}^{d}$ by means of a heuristic approximation, which was only recently justified by Brisard et al. [8], as summarized below.

We consider a linearly elastic heterogeneous material occupying the $d$-dimensional domain $\Omega$ characterized by its indicator function $\chi$

$$
\chi(\mathbf{x})= \begin{cases}1 & \text { if } \mathbf{x} \in \Omega, \\ 0 & \text { otherwise }\end{cases}
$$

For $\mathbf{x} \in \Omega, C(\mathbf{x})$ denotes the local elastic stiffness of the composite, while $\mathrm{C}_{0}$ denotes the (as yet unspecified) elastic stiffness of the so-called reference material.

\subsection{The modified Lippmann-Schwinger equation}

The modified Lippmann-Schwinger Equation (2) requires the fourth-order Green operator for strains $\Gamma_{0}^{\infty}$ of the unbounded domain $\mathbb{R}^{d}$, associated with the reference material $C_{0}$. In a prestressed, unbounded, homogeneous material with stiffness $C_{0}$, it relates the local strain to the applied (possibly inhomogeneous) prestress. A more precise definition of this operator can be found elsewhere (e.g. [4, 8-11]).

The following modified Lippmann-Schwinger equation is introduced [8], with unknown $\tau$ (the stress polarization), supported in $\Omega$

$$
\left(\mathrm{C}-\mathrm{C}_{0}\right)^{-1}: \tau+\Gamma_{0}^{\infty}[\tau-\chi \bar{\tau}]=\mathrm{E},
$$

where the loading parameter $\mathrm{E}$ is a symmetric, secondorder tensor. In the remainder of this paper, overlined quantities denote volume averages over the domain $\Omega$

$$
\bar{\tau}=\frac{1}{\operatorname{vol} \Omega} \int_{\mathbf{x} \in \Omega} \tau(\mathbf{x}) \mathrm{d} \mathbf{x} .
$$

From the solution $\tau$ to Equation (2), it is possible to construct a strain (resp. stress) field $\varepsilon$ (resp. $\sigma$ ) as follows

$$
\begin{aligned}
& \varepsilon=\mathrm{E}-\Gamma_{0}^{\infty}[\tau-\chi \bar{\tau}], \\
& \sigma=\mathrm{C}_{0}: \varepsilon+\tau=\mathrm{C}: \varepsilon,
\end{aligned}
$$

and it can be shown [8] that $\sigma$ thus constructed is divergence-free in $\Omega$ and that, provided the domain $\Omega$ is ellipsoidal, $\bar{\varepsilon}=\mathrm{E}$. In other words

1. the loading parameter $\mathrm{E}$ coincides with the macroscopic strain,

2. $\varepsilon$ is a compatible strain field,

3. $\sigma$ is an equilibrated stress field,

4. $\varepsilon$ and $\sigma$ are associated through the local constitutive law of the heterogeneous material.

Therefore, Equations (2) and (4) provide the solution to a new auxiliary problem (elastic equilibrium of the SVE) from which the apparent stiffness $C^{\text {app }}\left(C_{0}\right)$ can be defined

$$
\bar{\sigma}=\mathrm{C}^{\mathrm{app}}\left(\mathrm{C}_{0}\right): \bar{\varepsilon}=\mathrm{C}^{\mathrm{app}}\left(\mathrm{C}_{0}\right): \mathrm{E} .
$$

It should be noted that the apparent stiffness introduced above depends on the stiffness of the reference material, $\mathrm{C}_{0}$. It can be shown [8] that it is positive definite, and bounded from below (resp. above) by the apparent stiffness relating to static (resp. kinematic) uniform boundary conditions (defined in e.g. [12]). As a consequence, the apparent stiffness defined through Equation (5) is consistent in the homogenization sense: for statistically homogeneous and ergodic materials, it tends to the effective stiffness as the size of the domain $\Omega$ grows to infinity, regardless of the size of the $\operatorname{SVE} \Omega$.

\subsection{The principle of Hashin and Shtrikman}

For any trial field $\hat{\tau}$, the energy of Hashin and Shtrikman is defined as follows

$$
\mathcal{H}(\hat{\tau})=\overline{\hat{\tau}}: \mathrm{E}-\frac{1}{2} \overline{\hat{\tau}:\left(\mathrm{C}-\mathrm{C}_{0}\right)^{-1}: \hat{\tau}}-\frac{1}{2} \overline{\hat{\tau}: \Gamma_{0}^{\infty}[\hat{\tau}-\chi \overline{\hat{\tau}}]} .
$$

It can be shown [8] that the solution $\tau$ to the modified Lippmann-Schwinger Equation (2) is a critical point of $\mathcal{H}$. Furthermore

$$
\frac{1}{2} \mathrm{E}: \mathrm{C}^{\mathrm{app}}\left(\mathrm{C}_{0}\right): \mathrm{E}=\frac{1}{2} \mathrm{E}: \mathrm{C}_{0}: \mathrm{E}+\mathcal{H}(\tau) .
$$

The extremum principle of Hashin and Shtrikman can then be stated under further assumptions on the stiffness of the reference material

1. if $\mathrm{C}(\mathbf{x}) \leq \mathrm{C}_{0}$ for all $\mathbf{x} \in \Omega$, then $\mathcal{H}$ is minimal at $\tau$, and for any trial field $\hat{\tau}$

$$
\frac{1}{2} \mathrm{E}: \mathrm{C}^{\mathrm{app}}\left(\mathrm{C}_{0}\right): \mathrm{E} \leq \frac{1}{2} \mathrm{E}: \mathrm{C}_{0}: \mathrm{E}+\frac{1}{2} \mathcal{H}(\hat{\tau})
$$

2. if $\mathrm{C}(\mathbf{x}) \geq \mathrm{C}_{0}$ for all $\mathbf{x} \in \Omega$, then $\mathcal{H}$ is maximal at $\tau$, and for any trial field $\hat{\tau}$

$$
\frac{1}{2} \mathrm{E}: \mathrm{C}^{\mathrm{app}}\left(\mathrm{C}_{0}\right): \mathrm{E} \geq \frac{1}{2} \mathrm{E}: \mathrm{C}_{0}: \mathrm{E}+\mathcal{H}(\hat{\tau}),
$$

where inequalities between fourth-order tensors should be understood in the sense of the underlying quadratic forms. 


\subsection{The classical bounds of Hashin and Shtrikman}

In this section, and in the remainder of this paper, Greek indices always refer to material phases. Besides, random variables are indexed by $\omega$.

The celebrated bounds of Hashin and Shtrikman were initially derived in [5]; a more modern proof was proposed by Willis [4], who also considered the case of ellipsoidal distributions. Extension to ellipsoidal inclusions with different ellipsoidal distributions is due to Ponte Castañeda and Willis [13]. The random composite under consideration is made of $N$ linearly elastic, perfectly bounded phases. For $\alpha=1, \ldots, N$ and $\mathbf{x} \in \Omega, \chi_{\alpha}(\mathbf{x} ; \omega)$ denotes the indicator function at point $\mathbf{x}$ of phase $\alpha ; f_{\alpha}$ denotes the volume fraction of phase $\alpha: f_{\alpha}=\left\langle\chi_{\alpha}\right\rangle_{\omega}$ (where angle brackets denote ensemble averages). The local stiffness of the composite reads

$$
\mathrm{C}(\mathbf{x} ; \omega)=\sum_{\alpha=1}^{N} \chi_{\alpha}(\mathbf{x} ; \omega) \mathrm{C}_{\alpha},
$$

where $\mathrm{C}_{\alpha}$ denotes the stiffness of phase $\alpha$. To derive the bounds of Hashin and Shtrikman, the following trial field is selected

$$
\hat{\tau}(\mathbf{x} ; \omega)=\sum_{\alpha=1}^{N} \chi_{\alpha}(\mathbf{x} ; \omega) \hat{\tau}_{\alpha},
$$

where $\hat{\tau}_{1}, \ldots, \hat{\tau}_{N}$ are $N$ deterministic symmetric, secondorder tensors. Assuming that the reference medium is stiffer than all phases of the composite, Equation (8) gives

$$
\frac{1}{2} \mathrm{E}: \mathrm{C}^{\mathrm{app}}\left(\mathrm{C}_{0} ; \omega\right): \mathrm{E} \leq \frac{1}{2} \mathrm{E}: \mathrm{C}_{0}: \mathrm{E}+\mathcal{H}\left(\hat{\tau}_{1}, \ldots, \hat{\tau}_{N} ; \omega\right),
$$

where $\mathcal{H}\left(\hat{\tau}_{1}, \ldots, \hat{\tau}_{N} ; \omega\right)=\mathcal{H}(\hat{\tau} ; \omega)$ is a quadratic form of $\hat{\tau}_{1}, \ldots, \hat{\tau}_{N}$. Taking the ensemble average in Equation (12) and passing to the limit of infinite domains $\Omega$ leads to

$$
\frac{1}{2} \mathrm{E}: \mathrm{C}^{\mathrm{eff}}: \mathrm{E} \leq \frac{1}{2} \mathrm{E}: \mathrm{C}_{0}: \mathrm{E}+\left\langle\mathcal{H}\left(\hat{\tau}_{1}, \ldots, \hat{\tau}_{N} ; \omega\right)\right\rangle_{\omega},
$$

where $C^{\text {eff }}$ denotes the effective stiffness of the composite. The ensemble average $\left\langle\mathcal{H}\left(\hat{\tau}_{1}, \ldots, \hat{\tau}_{N} ; \omega\right)\right\rangle_{\omega}$ is a deterministic quadratic form of $\hat{\tau}_{1}, \ldots, \hat{\tau}_{N}$. It can be minimized with respect to these parameters, in order to produce the sharpest bounds on the effective stiffness in Equation (13). For a wide class of composites, the resulting bound can be computed explicitly [4, 13]; for isotropic composites, these bounds depend on the volume fraction and stiffness of each phase only.

\section{Towards improved bounds on the effective moduli?}

\subsection{Construction of enriched trial fields}

The trial field (11) considered by Hashin and Shtrikman [5] includes one point microstructural information only: the polarization stress at point $\mathbf{x} \in \Omega$ is totally defined by the phase at $\mathbf{x}$. Our aim in the present paper is to produce sharper bounds, by providing more microstructural information to the optimization process described in Section 2.3. In other words, we will consider an enrichment of the trial fields (11).

As already argued in Reference [2], the local volume fraction is a local descriptor of the microstructure which is believed to play a significant role on the macroscopic properties; we propose trial fields that incorporate this descriptor.

The local volume fraction is defined in this paper as the volume fraction of a specified phase contained in a sliding window of specified size. The present derivation is restricted to spherical windows of radius $a$. The local volume fraction of phase $\alpha$ at point $\mathbf{x} \in \Omega$ is the following quantity

$$
\tilde{f}_{\alpha}(\mathbf{x}, a ; \omega)=\frac{1}{W} \int_{\|\mathbf{y}\| \leq a} \chi_{\alpha}(\mathbf{x}+\mathbf{y} ; \omega) \mathrm{d} \mathbf{y},
$$

where $W$ denotes the volume of the spherical window. The local volume fraction is a random field; its expectation coincides with the global volume fraction $f_{\alpha}$. The $\tilde{f}_{\alpha}$ are linearly dependent; indeed, $\tilde{f}_{1}+\cdots+\tilde{f}_{N}=1$. As a consequence, only the $\tilde{f}_{1}, \ldots, \tilde{f}_{N-1}$ should be included in the proposed enriched trial field.

For the sake of simplicity, the remainder of this paper is restricted to two phase materials $(N=2)$. Therefore, the only local descriptor of the microstructure to be considered is the local volume fraction of phase 1 , which will be abusively called the local volume fraction, and denoted $\tilde{f}$; besides, the radius $a$ of the spherical window will also be dropped, so that we will write $\tilde{f}(\mathbf{x} ; \omega)$ rather than $\tilde{f}_{1}(\mathbf{x}, a ; \omega)$. We consider trial fields which are polynomials of the local volume fraction

$$
\hat{\tau}(\mathbf{x} ; \omega)=\sum_{\alpha=1}^{2} \sum_{k=0}^{p} \chi_{\alpha}(\mathbf{x} ; \omega) \tilde{f}(\mathbf{x} ; \omega)^{k} \hat{\tau}_{\alpha k},
$$

where $\hat{\tau}_{\alpha k}$ is a deterministic (symmetric) tensor. Obviously, the classical trial field (11) is retrieved with $p=0$; $p \geq 1$ effectively leads to an enrichment of the set of trial fields. In turn, this enrichment is expected to lead to sharper bounds on the effective properties of the microstructure.

\subsection{Evaluation of the energy of Hashin and Shtrikman}

Following the approach described in Section 2.3, we must evaluate the ensemble average of $\mathcal{H}$ for the trial field specified by Equation (15). Each term of $\mathcal{H}$ is evaluated separately below. Introducing the following moments of the local volume fraction

$$
Y_{\alpha k}(\mathbf{x})=\left\langle\chi_{\alpha}(\mathbf{x} ; \omega) \tilde{f}(\mathbf{x} ; \omega)^{k}\right\rangle_{\omega}
$$


it is readily verified that for statistically homogeneous materials, $Y_{\alpha k}$ does not depend on the observation point x. Indeed,

$$
\begin{aligned}
Y_{\alpha k}(\mathbf{x})= & \frac{1}{W^{k}}\left\langle\chi_{\alpha}(\mathbf{x} ; \omega) \prod_{i=1}^{k} \int_{\left\|\mathbf{y}_{i}\right\| \leq a} \chi_{1}\left(\mathbf{x}+\mathbf{y}_{i} ; \omega\right) \mathrm{d} \mathbf{y}_{i}\right\rangle_{\omega} \\
= & \frac{1}{W^{k}} \int_{\left\|\mathbf{y}_{1}\right\|, \ldots,\left\|\mathbf{y}_{k}\right\| \leq a}\left\langle\chi_{\alpha}(\mathbf{x} ; \omega) \prod_{i=1}^{k} \chi_{1}\left(\mathbf{x}+\mathbf{y}_{i} ; \omega\right)\right\rangle_{\omega} \\
& \times \mathrm{d} \mathbf{y}_{1} \cdots \mathrm{d} \mathbf{y}_{k},
\end{aligned}
$$

and the integrand in the last line does not depend on $\mathbf{x}$, due to statistical homogeneity. Evaluation of the first term of $\langle\mathcal{H}(\hat{\tau})\rangle$ is trivial

$$
\langle\hat{\tau}\rangle=\sum_{\alpha=1}^{2} \sum_{k=0}^{p} Y_{\alpha k} \hat{\tau}_{\alpha k}
$$

The second term of the ensemble-averaged energy of Hashin and Shtrikman reads

$$
\begin{aligned}
\frac{1}{2}\left\langle\overline{\left.\hat{\tau}:\left(\mathrm{C}-\mathrm{C}_{0}\right)^{-1}: \hat{\tau}\right\rangle}=\left\langle\frac{1}{2 V} \int_{\mathbf{x} \in \Omega} \sum_{\alpha, \beta, h, k}\right.\right. \\
\chi_{\alpha}(\mathbf{x} ; \omega) \chi_{\beta}(\mathbf{x} ; \omega) f(\mathbf{x} ; \omega)^{h+k} \\
\left.\hat{\tau}_{\alpha h}:\left[\mathrm{C}(\mathbf{x} ; \omega)-\mathrm{C}_{0}\right]^{-1}: \hat{\tau}_{\beta k} \mathrm{~d} \mathbf{x}\right\rangle_{\omega},
\end{aligned}
$$

where $V$ denotes the volume of the domain $\Omega$. Observing that $\chi_{\alpha}(\mathbf{x} ; \omega) \chi_{\beta}(\mathbf{x} ; \omega)=0$ for $\alpha \neq \beta$, and that $\chi_{\alpha}(\mathbf{x} ; \omega) \mathbf{C}(\mathbf{x} ; \omega)=\chi_{\alpha}(\mathbf{x} ; \omega) C_{\alpha}$ we finally find

$$
\overline{\left\langle\hat{\tau}:\left(\mathrm{C}-\mathrm{C}_{0}\right)^{-1}: \hat{\tau}\right\rangle}=\sum_{\alpha, h, k} Y_{\alpha, h+k} \hat{\tau}_{\alpha h}:\left(\mathrm{C}_{\alpha}-\mathrm{C}_{0}\right)^{-1}: \hat{\tau}_{\alpha k} .
$$

Evaluation of the last term is more complex ; first, application of the Green operator for strains is written as a convolution product

$$
\begin{aligned}
\overline{\hat{\tau}: \Gamma_{0}^{\infty}[\hat{\tau}-\chi \overline{\hat{\tau}}]} & =\frac{1}{V} \int_{\mathbf{x}, \mathbf{y} \in \Omega} \\
& \times \hat{\tau}(\mathbf{x}): \Gamma_{0}^{\infty}(\mathbf{y}-\mathbf{x}):[\hat{\tau}(\mathbf{y})-\overline{\hat{\tau}}] \mathrm{d} \mathbf{x} \mathrm{d} \mathbf{y},
\end{aligned}
$$

where the above integral should be understood in the sense of principal values (see e.g. [14]). Substituting in the above equation the general form (15) of the trial field, and taking the ensemble average leads to

$$
\begin{gathered}
\left\langle\overline{\hat{\tau}: \Gamma_{0}^{\infty} *(\hat{\tau}-\chi \overline{\hat{\tau}})}\right\rangle=\frac{1}{V} \sum_{\alpha, \beta, h, k} \int_{\mathbf{x}, \mathbf{y} \in \Omega} \\
\times\left[Z_{\alpha h, \beta k}(\mathbf{y}-\mathbf{x})-Y_{\alpha h} Y_{\beta k}\right] \hat{\tau}_{\alpha h}: \Gamma_{0}^{\infty}(\mathbf{y}-\mathbf{x}): \hat{\tau}_{\beta k} \mathrm{~d} \mathbf{x} \mathrm{d} \mathbf{y}
\end{gathered}
$$

where

$$
Z_{\alpha h, \beta k}(\mathbf{x}, \mathbf{y})=\left\langle\chi_{\alpha}(\mathbf{x} ; \omega) \chi_{\beta}(\mathbf{y} ; \omega)[\tilde{f}(\mathbf{x} ; \omega)]^{h}[\tilde{f}(\mathbf{y} ; \omega)]^{k}\right\rangle_{\omega} .
$$

The above statistical descriptor of the microstructure is translation-invariant $\left[Z_{\alpha h, \beta k}(\mathbf{x}, \mathbf{y})=Z_{\alpha h, \beta k}(\mathbf{y}-\mathbf{x})\right]$.
Further assuming that the microstructure is statistically isotropic, so that $Z_{\alpha h, \beta k}(\mathbf{x}, \mathbf{y})$ depends on the norm of $(\mathbf{y}-\mathbf{x})$ only $\left[Z_{\alpha h, \beta k}(\mathbf{x}, \mathbf{y})=Z_{\alpha h, \beta k}(\|\mathbf{y}-\mathbf{x}\|)\right]$, it can be shown that

$$
\begin{aligned}
\left\langle\overline{\hat{\tau}: \Gamma_{0}^{\infty}[\hat{\tau}-\chi \overline{\hat{\tau}}]}\right\rangle & =\sum_{\alpha, h, k} Y_{\alpha, h+k} \hat{\tau}_{\alpha h}: \mathrm{P}_{0}: \hat{\tau}_{\alpha k} \\
& -\sum_{\alpha, \beta, h, k} Y_{\alpha h} Y_{\beta k} \hat{\tau}_{\alpha h}: \mathrm{P}_{0}: \hat{\tau}_{\beta k},
\end{aligned}
$$

where $\mathrm{P}_{0}$ denotes the Hill tensor of a spherical inclusion embedded in the reference material $C_{0}$. Gathering Equations (17), (18) and (20) leads to the following expression of the ensemble averaged energy of Hashin and Shtrikman

$$
\begin{aligned}
\langle\mathcal{H}(\hat{\tau})\rangle= & \sum_{a, k} Y_{\alpha k} \hat{\tau}_{\alpha k}: \mathrm{E}-\frac{1}{2} \sum_{\alpha, h, k} Y_{\alpha, h+k} \\
& \hat{\tau}_{\alpha h}:\left[\left(\mathrm{C}_{\alpha}-\mathrm{C}_{0}\right)^{-1}+\mathrm{P}_{0}\right]: \hat{\tau}_{\alpha k} \\
& +\frac{1}{2} \sum_{\alpha, \beta, h, k} Y_{\alpha h} Y_{\beta k} \hat{\tau}_{\alpha h}: \mathrm{P}_{0}: \hat{\tau}_{\beta k} .
\end{aligned}
$$

\subsection{Determination of the optimum trial field}

Optimization of expression (21) with respect to $\hat{\tau}_{\alpha k}$ leads to the following characterization of the critical point

$$
\begin{aligned}
\sum_{k} Y_{\alpha, h+k}\left[\left(\mathrm{C}_{\alpha}-\mathrm{C}_{0}\right)^{-1}+\mathrm{P}_{0}\right]: \hat{\tau}_{\alpha k}=Y_{\alpha h} \\
\\
\left(\mathrm{E}+\sum_{\beta, k} Y_{\beta k} \mathrm{P}_{0}: \hat{\tau}_{\beta k}\right),
\end{aligned}
$$

for $\alpha=1,2$ and $h=1, \ldots, N$. The last term involves the ensemble average of the trial field $\hat{\tau}$ (see Eq. (17)), and we have

$$
\sum_{k} Y_{\alpha, h+k}\left[\left(\mathrm{C}_{\alpha}-\mathrm{C}_{0}\right)^{-1}+\mathrm{P}_{0}\right]: \hat{\tau}_{\alpha k}=Y_{\alpha h}\left(\mathrm{E}+\mathrm{P}_{0}:\langle\hat{\tau}\rangle\right),
$$

Introducing the inverse $X_{\alpha, h k}$ of $Y_{\alpha, h+k}$ in the following sense

$$
\sum_{\ell} X_{\alpha, h \ell} Y_{\alpha, \ell+k}=\sum_{\ell} Y_{\alpha, h+\ell} X_{\alpha, \ell k}=\delta_{h k},
$$

the solution to Equation (23) is readily found

$$
\begin{array}{r}
{\left[\left(\mathrm{C}_{\alpha}-\mathrm{C}_{0}\right)^{-1}+\mathrm{P}_{0}\right]: \hat{\tau}_{\alpha h}=\left(\sum_{k} X_{\alpha, h k} Y_{\alpha k}\right)} \\
\left(\mathrm{E}+\mathrm{P}_{0}:\langle\hat{\tau}\rangle\right) .
\end{array}
$$

Then, from Equation (24)

$$
\sum_{k} X_{\alpha, h k} Y_{\alpha k}=\sum_{k} X_{\alpha, h k} Y_{\alpha, k+0}=\delta_{h 0}
$$


which shows that $\hat{\tau}_{\alpha h}=0$ for $h \neq 0$, and the optimum trial field reduces to the classical form given by Equation (11). In other words, we get the surprising result that the enriched trial field (15) does not improve the classical Hashin and Shtrikman bounds on the effective elastic properties. This result is briefly extended to a wider class of enriched trial fields in Section 3.4 below.

\subsection{Extension to a wider class of trial fields}

It can be shown that the above results extend to a much wider class of trial fields. We consider here $n$ local descriptors of the microstructure $\phi_{1}(\mathbf{x} ; \omega), \ldots, \phi_{n}(\mathbf{x} ; \omega)$, and the following trial field

$$
\hat{\tau}(\mathbf{x} ; \omega)=\sum_{\alpha=1}^{N} \sum_{k=1}^{n} \chi_{\alpha}(\mathbf{x} ; \omega) \phi_{k}(\mathbf{x} ; \omega) \hat{\tau}_{\alpha k},
$$

where $\hat{\tau}_{\alpha k}$ is again a deterministic, second order, symmetric tensor. In order to ensure that Equation (25) is indeed an enrichment of Equation (11), we choose $\phi_{1}(\mathbf{x} ; \omega)=1$. It is assumed that these local descriptors of the microstructure are weakly isotropic in the sense that the following two-point statistical descriptors

$$
\left\langle\chi_{\alpha}(\mathbf{x} ; \omega) \phi_{h}(\mathbf{x} ; \omega) \chi_{\beta}(\mathbf{y} ; \omega) \phi_{k}(\mathbf{y} ; \omega)\right\rangle_{\omega}
$$

depend on the norm $\|\mathbf{y}-\mathbf{x}\|$ of the radius-vector only. Under this assumption, it can be shown that optimization of $\langle\mathcal{H}(\hat{\tau})\rangle$ with respect to $\hat{\tau}_{\alpha h}$ again leads to $\hat{\tau}_{\alpha h}=0$ for $h \neq 1$. This means that the classical bounds of Hashin and Shtrikman are again retrieved.

\section{Conclusion and outlook}

In this paper, we have presented an attempt at improving the classical bounds of Hashin and Shtrikman [5], by considering enriched trial fields which incorporate nontrivial local descriptors of the microstructure. By contrast, the only local descriptor used to derive the classical bounds is the phase at the observation point.

We first try to incorporate the local volume fractions as supplementary descriptors. This was suggested by previous work by Widjajakusuma et al. [2], and by the fact that this descriptor effectively introduces a length-scale (the size of the sliding window). We were therefore hoping to be able to produce bounds that would be sensitive to e.g. particle-size distributions (which is not the case of the classical bounds). However, our derivation shows that optimization of the ensemble-averaged energy of Hashin and Shtrikman again leads to the classical bounds. The supplementary descriptors are therefore totally ignored. This somewhat unexpected result was then extended to a very wide class of local descriptors of the microstructure.
Does this mean that improving the bounds of Hashin and Shtrikman is a hopeless task? Not necessarily. Indeed, the result presented in this paper is obtained under the assumption of isotropic probing of the microstructure (see Eq. (26)). In other words, it is assumed that the twopoint cross-correlations of all local descriptors only depend on the distance between the two observation points. This strongly suggests to use anisotropic probes; this will be investigated in future work.

\section{References}

[1] G.W. Milton, N. Phan-Thien, New bounds on effective elastic moduli of two-component materials, Proc. Royal Soc. London. Series A 380 (1982) 305-331

[2] J. Widjajakusuma, B. Biswal, R. Hilfer, Quantitative prediction of effective material properties of heterogeneous media, Comput. Mater. Sci. 16 (1999) 70-75

[3] Z. Hashin, S. Shtrikman, On some variational principles in anisotropic and nonhomogeneous elasticity, J. Mecha. Phys. Solids 10 (1962) 335-342

[4] J.R. Willis, Bounds and self-consistent estimates for the overall properties of anisotropic composites, J. Mech. Phys. Solids 2 (1977) 185-202

[5] Z. Hashin, S. Shtrikman, A variational approach to the theory of the elastic behaviour of polycrystals, J. Mech. Phys. Solids 10 (1962) 343-352

[6] C. Huet, Application of variational concepts to size effects in elastic heterogeneous bodies, J. Mech. Phys. Solids 38 (1990) 813-841

[7] M. Ostoja-Starzewski, Material spatial randomness: From statistical to representative volume element, Probabilistic Eng. Mech. 21 (2006) 112-132

[8] S. Brisard, K. Sab, L. Dormieux, New boundary conditions for the computation of the apparent stiffness of statistical volume elements, J. Mech. Phys. Solids 61 (2013) 2638-2658

[9] J. Korringa, Theory of elastic constants of heterogeneous media, J. Math. Phys. 14 (1973) 509-513

[10] R. Zeller, P.H. Dederichs, Elastic constants of polycrystals, Phys. Status Solidi B 55 (1973) 831-842

[11] E. Kröner, On the physics and mathematics of selfstresses. In: Topics in Applied Continuum Mechanics, edited by J.L. Zeman, F. Ziegler, Springer Verlag Wien, 1974, pp. 22-38

[12] T. Kanit, S. Forest, I. Galliet, V. Mounoury, D. Jeulin, Determination of the size of the representative volume element for random composites: statistical and numerical approach, Int. J. Solids Struct. 40 (2003) 3647-3679

[13] P. Ponte Castañeda, J.R. Willis, The effect of spatial distribution on the effective behavior of composite materials and cracked media, J. Mech. Phys. Solids 43 (1995) 1919 1951

[14] S. Torquato, Effective stiffness tensor of composite media-I, Exact series expansions, J. Mech. Phys. Solids 45 (1997) 1421-1448 